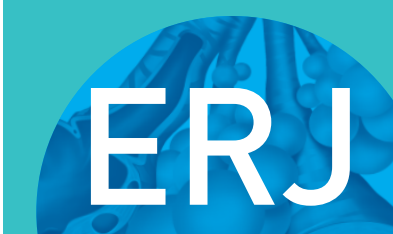

open research
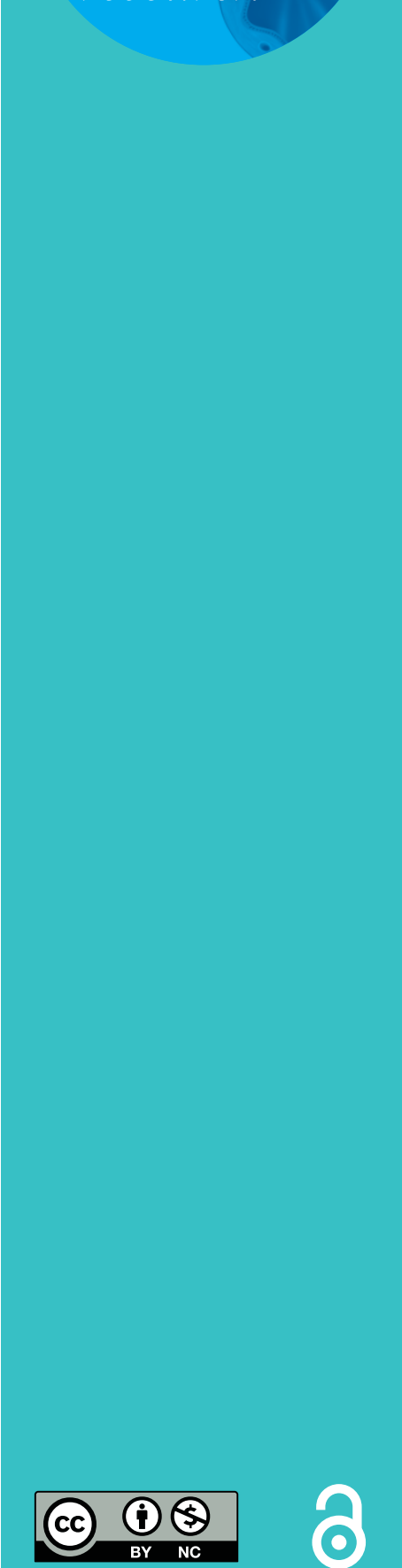

\section{EBUS-TBNA in PET-positive lymphadenopathies in treated cancer patients}

\author{
Juliana Guarize ${ }^{1}$, Monica Casiraghi ${ }^{1}$, Stefano Donghi ${ }^{1}$, Chiara Casadio ${ }^{2}$, \\ Cristina Diotti ${ }^{1}$, Niccolò Filippi ${ }^{1}$, Clementina Di Tonno ${ }^{2}$, Valeria Midolo ${ }^{2}$, \\ Patrick Maisonneuve ${ }^{3}$, Daniela Brambilla ${ }^{1}$, Chiara Maria Grana ${ }^{4}$, \\ Francesco Petrella ${ }^{1,5}$ and Lorenzo Spaggiari, ${ }^{1,5}$
}

Affiliations: 'Dept of Thoracic Surgery, European Institute of Oncology, Milan, Italy. ${ }^{2}$ Dept of Pathology, European Institute of Oncology, Milan, Italy. ${ }^{3}$ Dept of Epidemiology and Biostatistics, European Institute of Oncology, Milan, Italy. ${ }^{4}$ Dept of Nuclear Medicine, European Institute of Oncology, Milan, Italy. ${ }^{5}$ University of Milan, Dept of Haematology and Haematoncology, Milan, Italy.

Correspondence: Juliana Guarize, Dept of Thoracic Surgery, European Institute of Oncology, Via G. Ripamonti 435, 20141 Milan, Italy. E-mail: juliana.guarizedieo.it

ABSTRACT Mediastinal lymph node enlargement is common in the follow-up of patients with previously treated malignancies. The aim of this study is to assess the role of endobronchial ultrasound (EBUS) transbronchial needle aspiration (TBNA) for cyto-histological evaluation of positron emission tomography with ${ }^{18}$ fluorodeoxyglucose (PET) positive mediastinal and hilar lymph nodes developed in patients with previous malignancies.

All EBUS-TBNA cases performed from January 2012 to May 2016 were retrospective reviewed. Results of EBUS-TBNA in patients with mediastinal and/or hilar lymphadenopathies were analysed. Non-malignant cytopathologies were confirmed with surgical procedures or clinical and radiological follow-up.

Among 1780 patients, 176 were included in the analysis. 103 of these (58.5\%) had a diagnosis of tumour recurrence whereas 73 (41.5\%) had a different diagnosis: 63 (35.8\%) had a non-neoplastic diagnosis and 8 patients $(4.6 \%)$ had a different cell type malignancy. Samples were false-negative in $5(2.8 \%)$ out of 176 patients. The overall sensitivity, specificity, negative predicted value and diagnostic accuracy were $95.7 \%$ (95\% CI 90.2-98.6\%), 100\% (95\% CI 94.0-100\%), 92.3\% (95\% CI 83.2-96.7\%) and 97.2\% (95\% CI 93.5-98.8\%), respectively.

EBUS-TBNA demonstrated a pathological diagnosis different from the previous tumour in a large percentage of patients, confirming its strategic role in the management of patients with previously treated malignancies.

@ERSpublications

EBUS-TBNA changes the management of treated cancer patients http://ow.ly/vTnh30fBFaE

Cite this article as: Guarize J, Casiraghi M, Donghi S, et al. EBUS-TBNA in PET-positive lymphadenopathies in treated cancer patients. ERJ Open Res 2017; 3: 00009-2017 [https://doi.org/ 10.1183/23120541.00009-2017].

Received: Jan 162017 | Accepted after revision: Aug 252017

Conflict of interest: None declared.

Copyright $\odot$ ERS 2017. This article is open access and distributed under the terms of the Creative Commons Attribution Non-Commercial Licence 4.0. 


\section{Introduction}

Mediastinal lymph node enlargement at computed tomography (CT) scan is a common finding in the follow-up of patients with previously treated malignancies. Positron emission tomography with ${ }^{18}$ fluorodeoxyglucose (PET) scan evidence of increased uptake in mediastinal and hilar lymph nodes is generally suggestive of cancer recurrence, but histological confirmation is essential to plan the correct subsequent oncological strategy and avoid unnecessary treatment in the case of a non-malignant diagnosis.

For many years, invasive surgical procedures such as mediastinoscopy, video-assisted thoracoscopy (VATS) and thoracotomy were used for the diagnosis of intrathoracic adenopathy, depending on the lymph node location $[1,2]$.

Many recent articles have demonstrated the effectiveness and safety of endobronchial ultrasound (EBUS) transbronchial needle aspiration (TBNA) in different oncological settings [3]. As a minimally invasive procedure, EBUS-TBNA is currently the procedure of choice for mediastinal staging in lung cancer patients and tissue sampling for mediastinal and lung tumours, for the diagnosis of inflammatory disorders and also as the first approach in the diagnosis of lymphoproliferative disorders $[4,5]$. However, few data are available about the efficacy of EBUS-TBNA in the assessment of mediastinal and hilar lymph nodes in patients with previous (thoracic and extra thoracic) malignancy.

In this study, we aimed to assess the diagnostic yield of EBUS-TBNA for cyto-histological evaluation in a subset of patients treated for different malignancies who developed mediastinal and/or hilar lymph node enlargement during oncological follow-up.

\section{Methods}

This single-centre, retrospective study with prospective follow-up was approved by the Institutional Review Board and individual consent was obtained. All EBUS-TBNA procedures performed at the Interventional Pulmonology Unit of the Department of Thoracic Surgery at the European Institute of Oncology from January 2012 to May 2016 were identified. Clinical, radiological and pathological data were collected from clinical records. Prior to EBUS-TBNA procedures, all patients underwent non-invasive mediastinal and hilar evaluation with CT and PET scans. A lymph node was considered clinically suspect when its short axis was larger than $10 \mathrm{~mm}$ at CT scan and there was an increased pathological uptake at PET scan. When EBUS-TBNA did not provide a definitive diagnosis, patients underwent surgical histological confirmation or clinical and radiological follow-up.

Sensitivity, specificity, negative predictive value and diagnostic accuracy were calculated with the standard definition. False-negative cases were considered when the surgical procedure changed the final EBUS-TBNA diagnosis or there was clinical progression of disease during the follow-up observation period. In 2 patients, EBUS-TBNA samples were considered inadequate for diagnosis. Both patients were included as "false-negative" cases in the analysis of diagnostic performance.

EBUS-TBNA procedures were performed under local anaesthesia (1\% lidocaine) and moderate sedation was provided by an anaesthesiologist with spontaneous ventilation. All procedures were performed by the same team of interventional pulmonologists using a convex probe (EBUS Convex Probe BF-UC180F; Olympus) and a dedicated ultrasound processor (EU-ME1; Olympus). EBUS-TBNA specimens were collected with a 22-gauge dedicated needle (Vizishot NA-201SX-4022; Olympus).

A very small amount of the aspirated material was pushed out by the internal stylet and smeared onto glass slides for immediate on-site evaluation (rapid on site evaluation (ROSE)). Mirror slides were alcohol-fixed for posterior evaluation. Air-dried smears were immediately stained with a modified May-Grunwald Giemsa stain (MGG Quick Stain; Bio-Optica) and evaluated by the cytopathologist to confirm adequate tumour cells and/or lymph node material. The remaining aspirate and other needle passages were fixed in a formalin-like solution (Cytolyt; Hologic) for cell block processing and histological evaluation. Alcohol-fixed smears were stained with Papanicolaou and haematoxylin \& eosin stains.

During EBUS-TBNA procedures, in patients with a non-malignant diagnosis at ROSE, all lymphadenopathies with increased pathological PET scan uptake were sampled, and at least 1 mediastinal and 2 hilar stations were sampled when ROSE was negative for tumour cells and suspected for lymphadenitis granulomatosis.

Patients with negative EBUS-TBNA samples were referred for confirmatory surgical procedures after a multidisciplinary team discussion with thoracic surgeons, pulmonologists, radiologist, oncologists and radiotherapists when lymphadenopathies were considered highly suspicion for recurrence based on the CT and PET scan characteristics. 
The remaining patients with reactive lymph node samples underwent a mean of 20 months of clinical and radiological follow-up: 13 patients presented stability of the suspected lymph nodes, 9 patients presented a complete regression, and 1 patient presented progression of the disease and was included in the false-negative cases.

\section{Results}

During the study period, 1780 EBUS-TBNA procedures were performed and 176 were included in the analysis.

The patients' mean age was 62 years (range 31-87 years); 89 (50.6\%) were men and 87 (49.4\%) women. Patients' primary malignancies were breast cancer in 50 (28.4\%), lung in $46(26.1 \%)$, head and neck in $16(9.1 \%)$, genitourinary in $21(11.9 \%)$, female genital tract in $10(5.7 \%)$, gastrointestinal in $9(5.1 \%)$, lymphoproliferative disorders in 8 (4.6\%), multiple tumour sites in $12(6.8 \%)$ and other sites in 4 (2.3\%). Patient characteristics and primary malignancy sites are listed in table 1.

Overall 267 lymph node stations (200 mediastinal and 67 hilar) were sampled: station \#1 (n=1), station \#7 $(\mathrm{n}=101)$, \#2R $(\mathrm{n}=7)$, \#4R $(\mathrm{n}=75)$, \#10R $(\mathrm{n}=3)$, \#11R $(\mathrm{n}=23), \# 12 \mathrm{R}(\mathrm{n}=8)$, \#4L $(\mathrm{n}=16)$, \#10 ( $\mathrm{n}=1)$, \#L11 $(\mathrm{n}=24)$ and \#L12 (n=8) (table 2).

The final pathological diagnosis was related to the previous tumour in 103 (58.5\%) patients. Pathological diagnosis of malignant recurrences revealed breast cancer in 33 patients, lung cancer in 33 , head and neck cancer in 6, genitourinary tumours in 14 , female genital tract tumours in 4 , gastrointestinal cancer in 7 , lymphoproliferative disorders in 3 and other site tumours in 3 (1 thymoma, 1 adrenal and 1 melanoma) (table 3). The final diagnosis was different from the previous malignancy in 73 (41.5\%) patients, while pathological analysis disclosed a non-neoplastic condition in $63(35.8 \%)$ patients and a different cell type malignancy in 8 (4.6\%).

Among the 63 patients with a non-malignant diagnosis, EBUS-TBNA revealed a granulomatosis lymphadenitis compatible with sarcoidosis in 24 (38.1\%) patients, tuberculosis in 4 (6.3\%), necrotic lymph nodes in $2(3.2 \%)$ and reactive lymph nodes in $33(52.4 \%)$ patients.

\section{TABLE 1 Patient characteristics}



EBUS: endobronchial ultrasound; TBNA: transbronchial needle aspiration. 


\begin{tabular}{lcc} 
TABLE 2 Lymph node stations sampled by endobronchial ultrasound transbronchial needle \\
aspiration & & \\
Station & Description & Samples \\
\hline Total & & $267(100 \%)$ \\
Mediastinal & Lower paratracheal & $200(74.9 \%)$ \\
$4 \mathrm{~L}$ & Subcarinal & $16(6.0 \%)$ \\
7 & Highest mediastinal & $101(37.8 \%)$ \\
1 & Upper paratracheal & $1(0.4 \%)$ \\
$2 \mathrm{R}$ & Lower paratracheal & $7(2.6 \%)$ \\
$4 \mathrm{R}$ & & $75(28.1 \%)$ \\
Hilar & Hilar & $67(25.1 \%)$ \\
$10 \mathrm{~L}$ & Interlobar & $1(0.4 \%)$ \\
$11 \mathrm{~L}$ & Lobar & $24(9.0 \%)$ \\
$12 \mathrm{~L}$ & Hilar & $8(3.0 \%)$ \\
$10 \mathrm{R}$ & Interlobar & $3(1.1 \%)$ \\
$11 \mathrm{R}$ & Lobar & $23(8.6 \%)$ \\
$12 \mathrm{R}$ & & $8(3.0 \%)$ \\
\end{tabular}

A total of 14 patients underwent surgical procedures (mediastinoscopy or VATS) after EBUS-TBNA, with a definitive histological diagnosis of lymphadenitis granulomatosis in 6 patients, reactive lymph nodes in 5 and malignancy recurrence in 3. In 2 patients with EBUS-TBNA inadequate samples, mediastinoscopy revealed a reactive lymph node in 1 case and tumour recurrence in the other.

One patient with an EBUS-TBNA reactive lymph node sample had a progression of the disease during the clinical and radiological follow-up, resulting in 5 false-negative cases overall (figure 1).

Overall sensitivity, specificity, negative predicted value and diagnostic accuracy were 95.7\% (95\% CI 90.2-98.6\%), 100\% (95\% CI 94.0-100\%), 92.3\% (95\% CI 83.2-96.7\%) and 97.2\% (95\% CI 93.5-98.8\%), respectively (table 4 ).

\section{Discussion}

Mediastinal and hilar lymph node enlargement can be difficult to interpret in patients with previously treated malignancies. PET scan pathological glucose uptake usually raises suspicion of recurrence, but histological confirmation of neoplastic involvement is mandatory to plan the correct treatment.

Up to now, invasive surgical procedures (mediastinoscopy, VATS or thoracotomy) have been used for tissue confirmation in suspected lymph node recurrence. In our clinical practice, at the European Institute of Oncology, some oncologists do not approve the indication for such procedures because of its invasiveness, administering conservative (wait and see) or unnecessary treatments (chemotherapy) in case of suspected recurrence without histological confirmation.

Tissue sampling with non-surgical procedures such as endoscopic ultrasound-guided TBNA and EBUS-TBNA [6,7] has been used for mediastinal investigation after lung cancer treatment and extra-thoracic tumours [8] with good results.

TABLE 3 Endobronchial ultrasound transbronchial needle aspiration diagnosis of recurrence

\begin{tabular}{lc} 
Type of malignancy & Patients \\
\hline Total & $103(100 \%)$ \\
Breast & $33(32.0 \%)$ \\
Lung & $33(32.0 \%)$ \\
Genitourinary & $14(13.7 \%)$ \\
Head and neck & $6(5.9 \%)$ \\
Gastrointestinal & $7(6.9 \%)$ \\
Female genital tract & $4(3.9 \%)$ \\
Lymphoproliferative & $3(2.9 \%)$ \\
Other sites & $3(2.9 \%)$ \\
Thymoma & 1 \\
Adrenal & 1 \\
Melanoma & 1 \\
\hline
\end{tabular}




\section{TABLE 4 Endobronchial ultrasound (EBUS) diagnostic performance}

\begin{tabular}{|c|c|c|c|}
\hline & \multicolumn{2}{|c|}{ Final diagnosis" } & \multirow[t]{2}{*}{ Total } \\
\hline & Negative & Positive & \\
\hline EBUS negative & 60 & $5^{\Uparrow}$ & 65 \\
\hline EBUS positive & 0 & 111 & 111 \\
\hline Total & 60 & 116 & 176 \\
\hline \multicolumn{4}{|c|}{$\begin{array}{l}\text { Sensitivity=111/116=95.7\% }(95 \% \mathrm{Cl} 90.2-98.6 \%) \text {; specificity=60/60=100\% (95\% Cl } 94.0-100 \%) \text {; negative } \\
\text { predictive value }=60 / 65=92.3 \%(95 \% \mathrm{Cl} 83.2-96.7 \%) \text {; accuracy=171/176=97.2\% (95\% Cl } 93.5-98.8 \%) \text {. } \\
\# \text { : based on the results of mediastinoscopy/video-assisted thoracoscopy and/or clinical and radiological } \\
\text { follow-up; } 7 \text { : four patients underwent surgical histological confirmation and one patient had clinical/ } \\
\text { radiological progression of the disease. }\end{array}$} \\
\hline
\end{tabular}

Our current study demonstrated that EBUS-TBNA was useful and safe in mediastinal and hilar lymph node evaluation in patients with previously treated (thoracic and extra-thoracic) malignancies with no related complications. There are some important findings in the study. We found that 60 patients (34.1\%) had a non-neoplastic disease and 24 of them (40.0\%) had a non-necrotising granulomatosis lymphadenitis identified with EBUS-TBNA samples. Out of 24 patients, 2 underwent a surgical procedure (1 mediastinoscopy and 1 VATS) with histological confirmation of sarcoidosis. In 4 out of 60 patients (6.7\%), EBUS-TBNA revealed a necrotising granulomatosis lymphadenitis with a lymph node culture sample positive for Mycobacterium tuberculosis complex. Of those 4 patients, 2 underwent mediastinoscopy, with pathological confirmation of tuberculosis lymphadenitis.

In 2 patients (3.3\%), EBUS-TBNA revealed necrotic lymph node samples. Both patients had only 1 PET-positive lymph node station and were addressed to follow-up. One patient had complete regression of the lymph node and the other remained stable after 20 months of follow-up.

Our results are similar to those of other reports showing that the most common EBUS-TBNA non-neoplastic finding was non-necrotising granulomatosis and sarcoid reaction in that cohort of patients [8].

We also found that 103 patients (58.5\%) had a lymph node recurrence of a previous malignancy. Recurrence was related to breast in $33(32.0 \%)$ and lung cancer in 33 patients $(32.0 \%)$. In breast cancer patients, EBUS-TBNA provided adequate samples for genetic assessment, indicating possible variations in cell hormonal receptors guiding subsequent therapy.

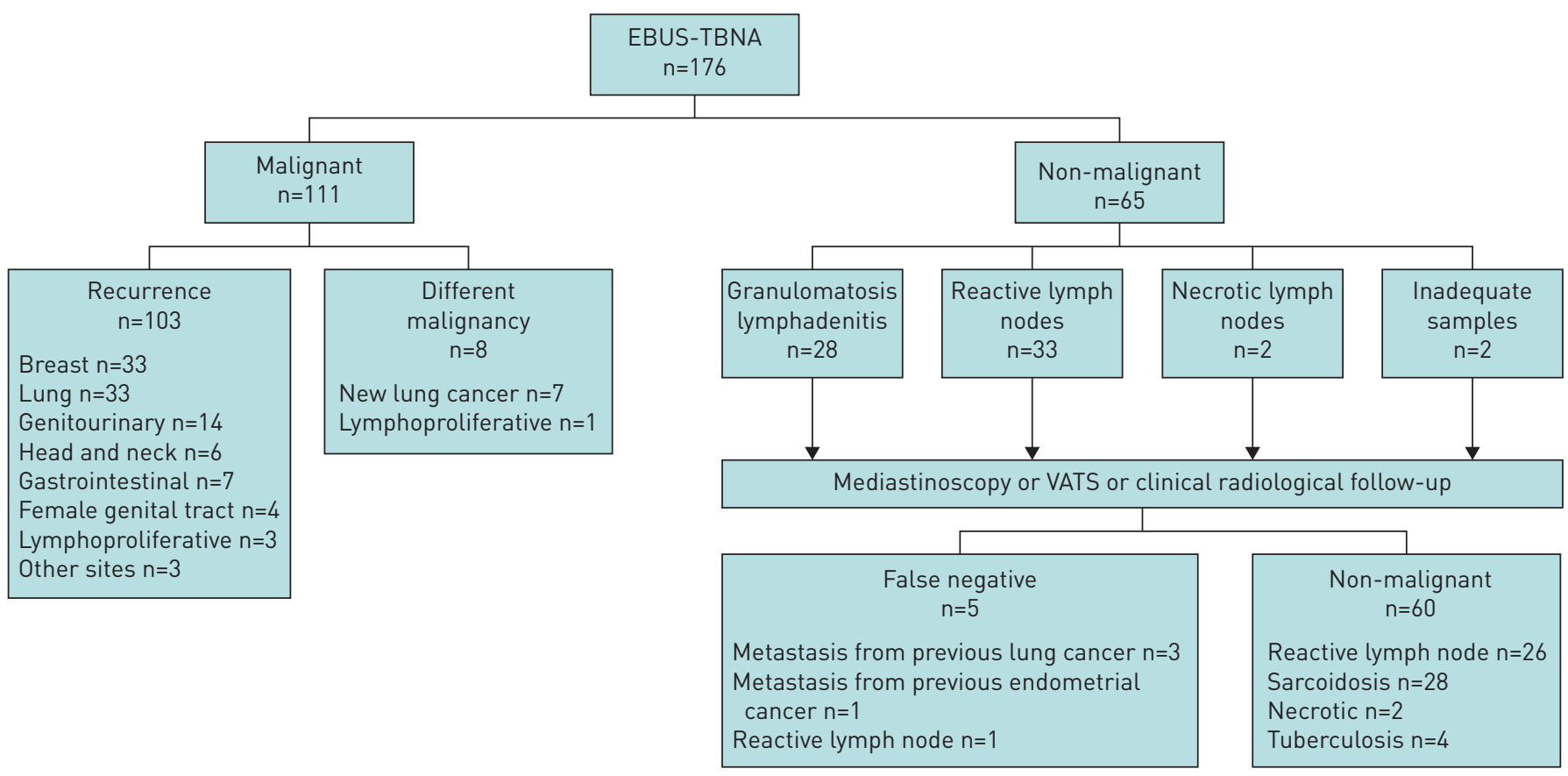

FIGURE 1 Flowchart of patients. EBUS: endobronchial ultrasound; TBNA: transbronchial needle aspiration. 
In lung cancer recurrence, EBUS-TBNA was able to provide the tumour subtype in all patients. In 16 patients with lung adenocarcinoma recurrence, EBUS-TBNA allowed the determination of epidermal growth factor receptor (EGFR) and anaplastic lymphoma kinase (ALK) genetic mutations. This finding is in agreement with our previously reported experience with genetic assessment with EBUS-TBNA samples, demonstrating that EBUS-TBNA provides adequate material for molecular analyses in a similar percentage of cases when compared to surgical specimens [9].

Finally, we found that 8 patients $(4.6 \%)$ had a different neoplasm from the previous tumour: 7 patients had a second primary lung cancer and 1 patient a lymphoproliferative disorder diagnosed with EBUS-TBNA samples and confirmed with mediastinoscopy for further lymphoma subtyping.

Other studies recently founded similar results [10]. That study reported that EBUS-TBNA demonstrated a second primary lung cancer in $25 \%$ of 44 patients with a previous lung cancer.

In our cohort, the vast majority of patients did not have pulmonary lesions.

From the 7 patients with a new lung cancer, 4 patients presented a single pulmonary lesion and multiple lymph nodes with PET pathological uptake. In the remaining 3 patients, 1 patient with a previous pneumonectomy presented a pleural lesion and a subcarinal lymph node, 1 patient presented only a peribronchial tissue and subcarinal lymph node without pulmonary lesions, and the remaining patient presented only multiple bilateral lymph node stations.

In our cohort of patients, EBUS-TBNA findings determined the subsequent treatment and prognosis of patients, preventing delayed therapy in cases of disease recurrence and unnecessary therapy in non-neoplastic conditions.

We recognise the limitations of our study. Not all patients underwent a surgical procedure to confirm the negativity of EBUS-TBNA samples. In 3 out of 14 (21.4\%) patients who underwent mediastinoscopy or VATS after EBUS-TBNA, a malignant histology was revealed after the surgical procedure. These data confirm the need for a surgical invasive investigation when EBUS-TBNA reveals a non-neoplastic diagnosis in highly suspect cases in this subset of patients.

In conclusion, EBUS-TBNA is able to provide a definitive diagnosis in cases of mediastinal and hilar lymphadenopathy suspected to be tumour recurrence in patients with previously treated malignancies, clarifying the nature of lymphadenopathies in cases of non-neoplastic disorders and guiding subsequent therapy in cases of recurrence.

Given its safety and low invasiveness, it should be used as first approach in this subset of patients, even if surgical histological confirmation should be performed in highly suspicion cases.

\section{Acknowledgements}

This study was presented at the ERS International Congress 2016 [11].

\section{References}

1 Hammoud ZT, Anderson RC, Meyers BF, et al. The current role of mediastinoscopy in the evaluation of thoracic disease. J Thorac Cardiovasc Surg 1999; 118: 894-899.

2 Ginsberg RJ. Evaluation of the mediastinum by invasive techniques. Surg Clin North Am 1987; 67: 1025-1035.

3 Varela-Lema L, Fernández-Villar A, Ruano-Ravina A. Effectiveness and safety of endobronchial ultrasound-transbronchial needle aspiration: a systematic review. Eur Respir J 2009; 33: 1156-1164.

4 Kinsey CM, Arenberg DA. Endobronchial ultrasound-guided transbronchial needle aspiration for non-small cell lung cancer staging. Am J Respir Crit Care Med 2014; 189: 640-649.

5 Wahidi MM, Herth F, Yasufuku K, et al. Technical aspects of endobronchial ultrasound-guided transbronchial needle aspiration: CHEST Guideline and Expert Panel Report. Chest 2016; 149: 816-835.

6 Stigt JA, Oostdijk AH, Timmer PR, et al. Comparison of EUS-guided fine needle aspiration and integrated PET-CT in restaging after treatment for locally advanced non-small cell lung cancer. Lung Cancer 2009; 66: 198-204.

7 Herth FJ, Annema JT, Eberhardt R, et al. Endobronchial ultrasound with transbronchial needle aspiration for restaging the mediastinum in lung cancer. J Clin Oncol 2008; 26: 3346-3350.

8 Navani N, Nankivell M, Woolhouse I, et al. Endobronchial ultrasound-guided transbronchial needle aspiration for the diagnosis of intrathoracic lymphadenopathy in patients with extrathoracic malignancy: a multicenter study. J Thorac Oncol 2011; 6: 1505-1509.

9 Casadio C, Guarize J, Donghi S, et al. Molecular testing for targeted therapy in advanced non-small cell lung cancer: suitability of endobronchial ultrasound transbronchial needle aspiration. Am J Clin Pathol 2015; 144: 629-634.

10 Anraku M, Pierre AF, Nakajima T, et al. Endobronchial ultrasound-guided transbronchial needle aspiration in the management of previously treated lung cancer. Ann Thorac Surg 2011; 92: 251-255.

11 Guarize J, Magni E, Casadio T, et al. EBUS-TBNA in positron emission tomography (PET) positive mediastinal lymph nodes in previously radically treated malignancies. Eur Respir J 2016; 48: Suppl. 60, PA2043. 